\title{
The Design and Analysis of Remote Image Monitoring System for Electric Power Automation Based on CDMA
}

\author{
ZHOU Zhengjie ${ }^{1, \text { a }}$ \\ ${ }^{1}$ Guangxi Technological College of Machinery and Electricity, Nanning Guangxi, 530007 \\ azhouzhengjie@126.com
}

Keywords: CDMA; Remote Image Monitoring; Electric Power Automation

\begin{abstract}
With the development of codec technology and wireless broadband access system of the opening, use them as a hosted network, for low bit rate wireless video monitoring system is more and more get the attention of people. Based on CDMA network of the Internet are introduced in detail based on CDMA wireless communication for the working principle of the image monitoring and designed to CDMA mode of private network can be applied to power plant water station in remote areas, such as image monitoring scheme, involving network mode, the system monitoring module unit interior, related compression algorithm and background removal algorithm and should have the function of the system the background, proposed to improve the development direction of the wireless monitoring system. Along with the increasingly mature CDMA remote image monitoring system, remote monitoring of power system automation will inject fresh blood, to make contribution to the improvement of electric power automation.
\end{abstract}

\section{Introduction}

With the continuous development of electric power automation technology, make the power system automation level gradually improve, power plants, substations, stations and so on all need by monitoring system gradually achieve less people, no regulation, in order to improve production efficiency. Remote image monitoring is the telemetry, remote and remote control of further added. More and more remote image monitoring system of the electric power enterprises as a new means of power system automation management [1]. Remote power monitoring system was built in the center of the electric power dispatching management monitoring center, set up in each substation video, audio, data acquisition unit, or in power plants, substations, etc to set up the monitoring center on their equipment, video surveillance equipment and working environment [2]. Monitoring center of the data of each monitoring unit image monitoring and surveillance, can real-time, accurate, and directly understand and master the situation of the monitoring unit, and respond to what is happening in time, and can carry on the storage to the related data, analysis, provides forceful support for later decisions and to improve, to adapt to the needs of the modernization development.

In this paper, which used in power system communication channel are analyzed and compared, combined with the rapid development of communication technology, put forward a kind of image monitoring system based on CDMA wireless channel. Based on the analysis of the characteristics of the CDMA structure and, fully affirmed the emerging transmission medium is applied to power system automation feasibility and broad application prospect, thus proves the system to improve the power system automation, improve the reliability and real-time performance is of great significance. CDMA network for electric power automation system provides a fast and reliable, easy extension, and easy to maintain communication mode, and the remote monitoring system based on the wireless communication, has no wiring work, reduce the network construction cost, save a lot of cost, short construction period, the expansion of sex is strong, high reliability, fast transfer rate, numerous advantages of rapidity, etc. As long as it is covered in CDMA network, image monitoring can at any time and do the real mobile monitoring. 


\section{CDMA communication technology overview}

Simulation system USES DFMA (Frequency Division Multiple Access) technology, GMS uses TDMA technology, CDMA is also known as Code Division Multiple Access, CDMA is required for the modern mobile communication network of the large capacity, high quality, comprehensive business, soft switch, international roaming and so on requirement and design of a mobile communications technology [3]. CDMA is based on the spread spectrum technology, the need to send a certain signal bandwidth information data, using a high-speed bandwidth than the signal bandwidth pseudorandom Code modulation, make original data signal bandwidth is extended, the carrier modulation and send out again. Receive comprised use identical pseudo random Code, and the bandwidth of the signal correlation processing, the broadband signal into the original data of narrow-band signals that the expansion, in order to realize the information communication [4]. In CDMA wireless network and the Internet as a channel of communication model is shown in figure 1.

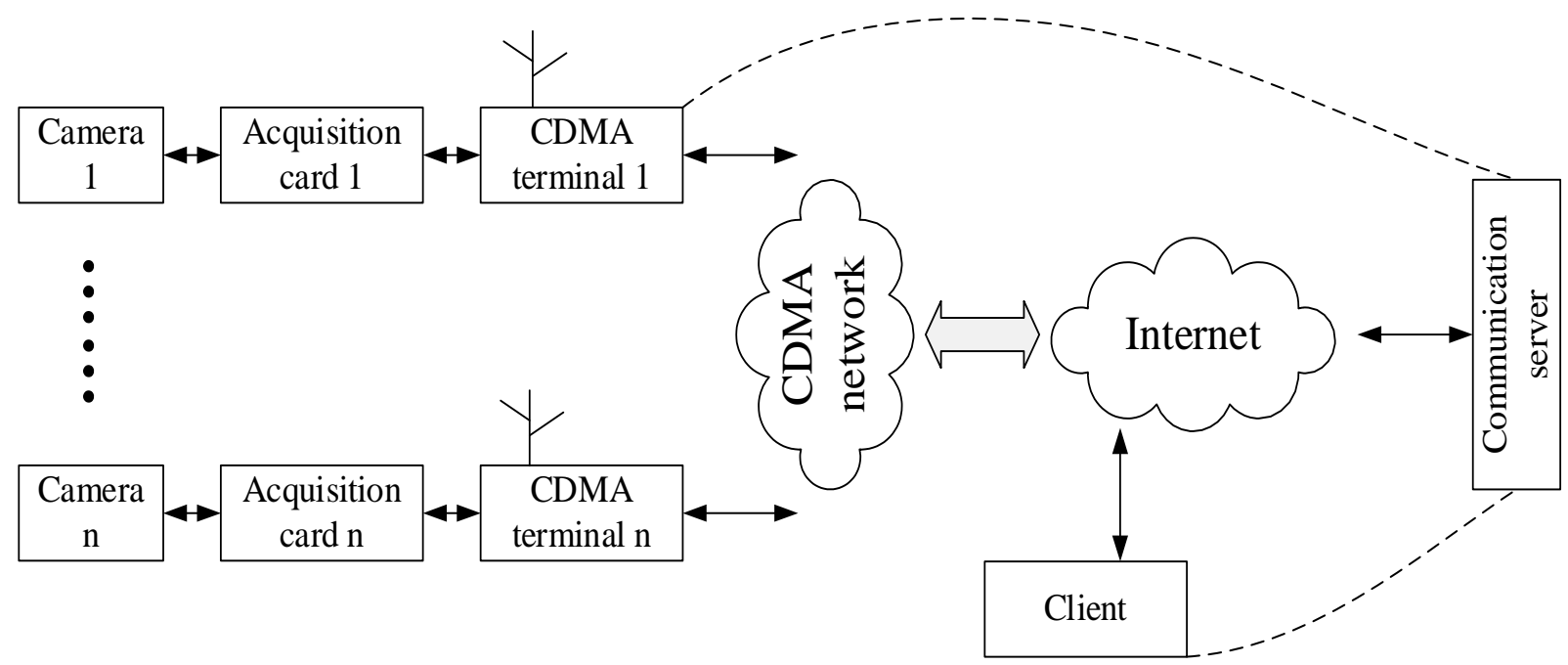

Figure 1. CDMA communication model

This system to monitor the unit's LAN is a network platform, based on the TCP/IP network communication protocol. Any one terminal image of system can be based on their authority to monitor the front camera [5]. In the system, the front camera is a camera to DCMA terminal image transmission, again by the end of CDMA and Internet network to the communication server uplink process" from the camera video signal acquisition card sampling and compression coding, form a MPEG 4 standard image data stream, CDMA terminals through RS232 input, PI again in the CDMA terminal through the CDMA network packet way, with the user name and password are card way of dial-up access to the Internet, send connected to the network communication server or client machine, unpack and decompression separated by its former video image signal and display on the display.

\section{Structure analysis of remote image monitoring system for electric power automation based on CDMA}

In this article, the design of system is an embedded general video monitoring system based on TMS320DM642, with multiple extensions interface, can be used according to actual needs and the flexibility to choose the suitable environment interface, can realize real-time multi-channel video acquisition, and to support complex audio video compression algorithm. The structure of the whole system is shown in figure 2 . 


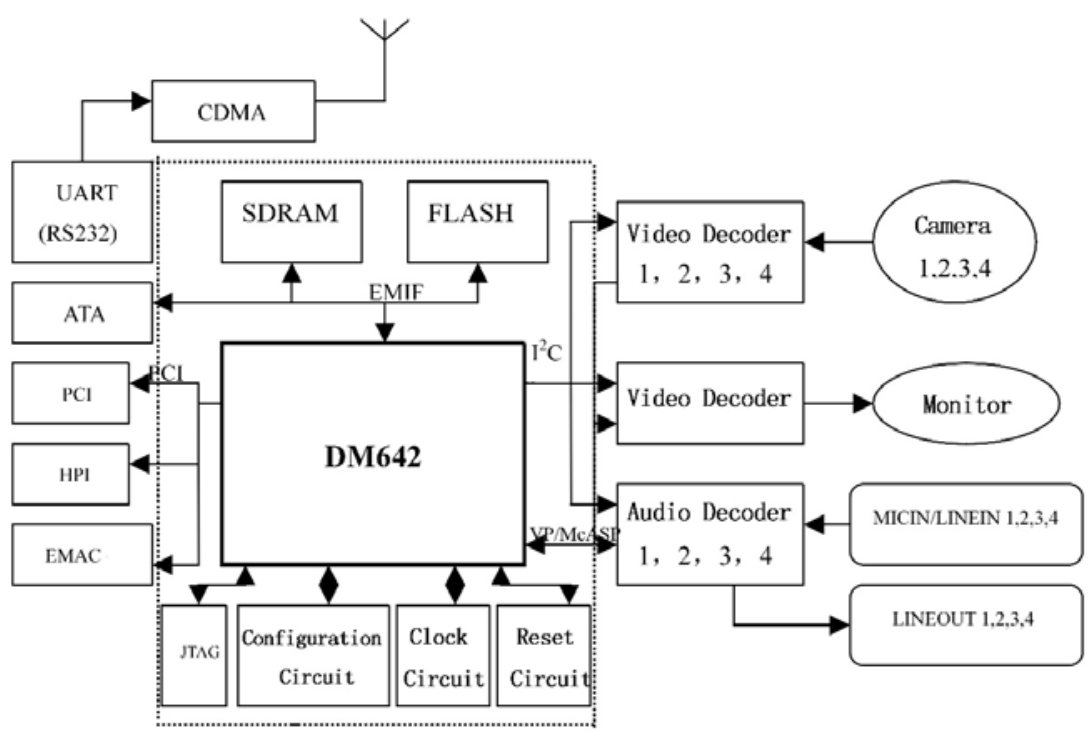

Figure 2. Structure of remote image monitoring system for electric power automation based on CDMA

DM642 video port through four video decoding chip TVP5150A can easily realize the seamless connection and four CMOS camera. Camera output analog video signal after TVP5150A modulus conversion, formation of BT. 656 format digital video signal, from the DM642 video port input, the software encoder based on DM642 coding compression processing, processing of digital video stream data by extending the ATA interface to transfer data to local storage IDE hard disk; (2) the data package and then through the Ethernet interface to transfer data to the remote server to save. Audio digital, analog signal through the audio codec into DM642 handled or preserved. UART interface from the EMIF extension, via a standard RS232 interface system working condition can be visualized in PC super terminal window, immediately to facilitate designers working condition of the system. This project is to use the RS232 serial port input video streaming data into CDMA module, and then sent to the remote monitoring terminal.

\section{Overall design of remote image monitoring system for electric power automation based on CDMA}

With the constant improvement of the power system automation level, the substation, substation, stations and so on to achieve less people or unattended, in order to improve production efficiency. Rely on the original manual methods cannot meet the needs of the electric power monitoring and control system development. Go on a remote video monitoring is gradually. Remote video monitor system can monitor and record the operation situation of substation and the safety of the equipment, monitoring of power equipment and operation, timely detection and processing accident, help to improve the safety and reliability of power system automation, and provides the later analysis of the information about the image of the accident. At the same time it also has a fire prevention, guard against theft, and other functions. Substation image monitoring is one of the typical image monitoring power system application. Here, in power system transformer substation, for example, based on CDMA remote image monitoring system of electric power automation design, the system block diagram is shown in figure 3. This paper is divided into three parts, the overall scheme of the front-end equipment system, the network transmission and remote monitoring center.

Front-end equipment system. Mainly refers to the image acquisition and processing power equipment, equipment mainly includes the camera system, infrared smoke alarm, video encoding server, temperature, humidity sensor and solar energy power supply system, including remote monitoring front-end hardware equipment and control equipment.

Remote network transmission. It is composed of communication network, data center and data terminal. Transmission of the communication network will use the bus network. Bus network 
using the cable transmission on hardware equipment, but high-speed network communication link equipment can be completely together, even if some equipment failed to remove the equipment, does not affect the work of other devices.

Monitoring center system. Its role in the electric power monitoring and control system is to be reckoned with, but not set control center part of the electric power monitoring and control system, or monitoring the management system and monitoring the main image of the customer terminal monitoring server. It mainly completed the following functions, such as the image decoding and receiving, the management of electric power remote monitoring power monitoring of priority, the distribution of the electric power remote control signal coordination, remote real-time monitoring and video image video playback, backup, restore, etc.

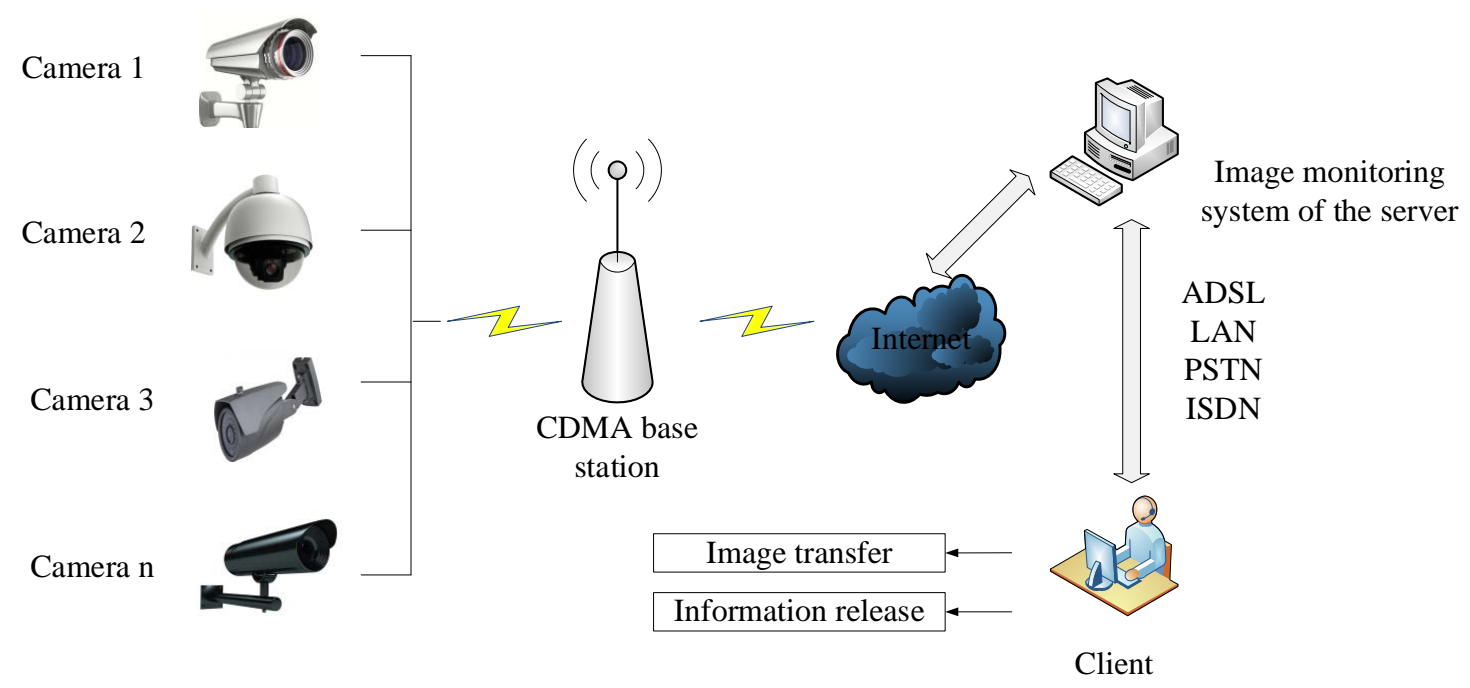

Figure 3.Hardware frame of remote image monitoring system for electric power automation based on CDMA

\section{Conclusion}

Electrical equipment remote monitoring is a complex issue, involving the construction of a communication system, the information acquisition, power supply equipment and image processing, such as designing monitoring systems more difficult. This study proposes a remote power equipment monitoring system based on CDMA network design scheme, the processed data to CDMA network terminal, transform information into digital signal launch out, central control center to receive information, through image processing, automatic detection of abnormal state of the transmission line environmental conditions, and call the police, have solved the problem of video remote collection and transmission. And provides the function of the camera video information collection. When electric power equipment failure or abnormal operation, the monitoring center can alarm and optimization in a timely manner, and ensure the normal operation of the transmission line.

\section{References}

[1] Wang X, Li H, Cong L: Power System Technology, 2009, 18: 038.

[2] Zhu X, Li D, He D: Computers and Electronics in Agriculture, 2010, 71: S3-S9.

[3] Wang W, Xu Y, Khanna M: Computer Networks, 2011, 55(15): 3604-3629.

[4] Zidao M, Zhengbing Z: Computer Measurement \& Control, 2011, 2: 070.

[5] Xin D U: Telecommunications for Electric Power System, 2011, 8: 010. 